\section{Rate of cervical cancer, severe intraepithelial neoplasia, and adenocarcinoma in situ in primary HPV DNA screening with cytology triage: randomised study within organised screening programme}

\author{
Ahti Anttila, research director of the mass screening registry, ${ }^{1}$ Laura Kotaniemi-Talonen, researcher, ${ }^{1}$ Maarit \\ Leinonen, researcher, ${ }^{1}$ Matti Hakama, professor, ${ }^{1}$ Pekka Laurila, chief physician, ${ }^{2}$ Jussi Tarkkanen, senior \\ consultant in pathology, ${ }^{2}$ Nea Malila, director of the mass screening registry, professor, ${ }^{1,3}$ Pekka Nieminen, \\ chief physician ${ }^{4,5}$
}

\begin{abstract}
'Mass Screening Registry of the Finnish Cancer Registry, Pieni Roobertinkatu 9, FIN-00130 Helsinki, Finland

${ }^{2}$ HUSLAB Kätilöopisto Pathology Laboratory, Helsinki

${ }^{3}$ School of Public Health, University of Tampere, Tampere

${ }^{4}$ Department of Obstetrics and Gynaecology, Helsinki University Central Hospital, Helsinki

${ }^{5}$ Pathology Laboratory of the Finnish Cancer Society, Helsinki Correspondence to: A Anttila ahti.anttila@cancer.fi

Cite this as: $B M J$ 2010;340:c1804 doi:10.1136/bmj.c1804
\end{abstract}

\section{ABSTRACT}

Objective To assess the performance and impact of primary human papillomavirus (HPV) DNA screening with cytology triage compared with conventional cytology on cervical cancer and severe pre-cancerous lesions. Design Randomised trial.

Setting Population based screening programme for cervical cancer in southern Finland in 2003-5.

Participants 58076 women, aged 30-60, invited to the routine population based screening programme for cervical cancer.

Interventions Primary HPV DNA test (hybrid capture II) with cytology triage if the result was positive or conventional cytological screening (reference).

Main outcome measures Rate of cervical cancer, cervical intraepithelial neoplasia (CIN) grade III, and adenocarcinoma in situ (as a composite outcome referred to as CIN III+) during 2003-7 through record linkage between files from the screening registry and the national cancer registry.

Results In the HPV and conventional arms there were 95600 and 95700 woman years of follow-up and 76 and 53 cases of CIN III+, respectively (of which six and eight were cervical cancers). The relative rate of CIN III+ in the HPV arm versus the conventional arm was 1.44 (95\% confidence interval 1.01 to 2.05$)$ among all women invited for screening and 1.77 (1.16 to 2.74) among those who attended. Among women with a normal or negative test result, the relative rate of subsequent CIN III+ was 0.28 (0.04 to 1.17). The rate of cervical cancer between arms was 0.75 ( 0.25 to 2.16 ) among women invited for screening and 1.98 (0.52 to 9.38) among those who attended.

Conclusions When incorporated into a well established organised screening programme, primary HPV screening with cytology triage was more sensitive than conventional cytology in detecting CIN III+ lesions. The number of cases of cervical cancer was small, but considering the high probability of progression of CIN III the findings are of importance regarding cancer prevention.
Trial registration Current Controlled Trials ISRCTN23885553.

\section{INTRODUCTION}

The incidence of invasive cancer is the most informative standard in the evaluation of cervical cancer screening programmes and screening methods. Data on cancer incidence and mortality, however, are available for only some of the conventional cervical smear based screening programmes. ${ }^{12}$ Alternative technologies such as liquid based cytology or human papillomavirus (HPV) screening or vaccination have been proposed as possible means to improve prevention of cervical cancer. ${ }^{23}$ Most studies have relied on surrogate end points such as rates of cervical intraepithelial neoplasia (CIN) or sometimes only cytological findings. ${ }^{4}$ These end points are highly variable between programmes and age groups and do not necessarily precisely represent the real effect on incidence and mortality. ${ }^{25}$ Longitudinal information on the most severe surrogate endpoint markers ${ }^{6-9}$ or on the incidence of cervical cancer and mortality ${ }^{10}$ is rarely available for the newer methods.

We evaluated the impact of primary HPV DNA screening with cytology triage on cervical cancer, severe cervical intraepithelial neoplasia, or adenocarcinoma in situ (as a composite outcome referred to as CIN III+). We used a randomised design incorporated in the routine population based organised screening programme for cervical cancer in Finland. The reference test was conventional cytology. The evaluation was based on the total number of cases of CIN III+ detected within five years after the index invitation.

\section{METHODS}

\section{Recruitment}

The study was based on follow-up during 2003-7 in women randomised $(1: 1)$ to primary HPV DNA screening with cytology triage or conventional 
cytological screening within the population based screening programme for cervical cancer in southern Finland between 1 January 2003 and 31 December 2005. We used data from eight municipalities and two screening laboratories. In total 58282 women were invited to participate in the screening programme (fig 1). The screening invitations at the recruitment phase included the five yearly invitations to the screening programme. Information on study design, screening methods used, and cross sectional screening findings have been previously published. ${ }^{11-14}$

\section{Randomisation and exclusions}

Of the 58282 women, 29144 (50\%) were individually randomised to screening with the HPV DNA test and 29138 to conventional cytological screening as the control arm. Randomisation was done two to 12 months before invitation. After randomisation, 206 women were excluded, 107 from the HPV arm and 99 from the conventional arm (fig 1). We included data from 58076 women in the analysis.

\section{Conventional cytology screening protocol}

The national screening programme for cervical cancer in Finland was started in the early 1960s, and women aged 30-60 are invited by letter to attend screening every five years. Trained nurses or midwives usually take screening samples in primary healthcare facilities in the municipality. In the trial, cytological sampling was the same in both arms: a VCE smear (vaginal,

Enrolment

Women eligible for routine screening in 2003-5 ( $n=58$ 282)

\begin{tabular}{l} 
Allocation \\
Randomisation to HPV screening \\
(experimental arm) ( $\mathrm{n}=29144)$ \\
Excluded from analysis ( $\mathrm{n}=107)$ : \\
Died before invitation ( $\mathrm{n}=31$ ) \\
Emigration before invitation ( $\mathrm{n}=41$ ) \\
Earlier cervical cancer ( $\mathrm{n}=35$ ), diagnosed at \\
any time since 1953 \\
Analysis \\
Valid invitations ( $\mathrm{n}=29$ 037) \\
19449 attended and 9588 did not attend \\
(17 795 (91.5\% of attenders) underwent HPV \\
test and 1654 women (8.5\%) underwent \\
conventional cytology as primary screening test) \\
Follow-up \\
76 cases of $\geq$ CIN III observed after invitation, \\
59 among attendees and 17 among \\
non-attendees. Among attendees 56 cases \\
identified after HPV test and 3 cases identified \\
after cytology as primary screening test. None \\
lost to follow-up (all invitees under register \\
based follow-up until death, emigration, \\
diagnosis of cervical cancer, or end of follow-up) \\
(222 emigrated (censored at emigration) and \\
213 died from causes other than cervical cancer \\
during follow-up) \\
\hline
\end{tabular}

Fig 1| Enrolment, random allocation, completeness of follow-up, and analysis cervical and endocervical samples) was taken with a cytobrush from the junction and endocervix, and with two spatulas from the ectocervix and vaginal fornices, and spread on the same objective glass. The fixative was 95\% ethylalcohol and Papanicolaou staining was used. A cytotechnician reviewed all the screening smears and referred any abnormal findings to a cytopathologist. The cytopathologist also re-evaluated 10$20 \%$ of the smears with normal results. The cytopathologist was responsible for the final diagnosis and recommendations.

In conventional practice, a referral for colposcopy and biopsy is based on a cytology result equal to Papanicolaou class III-V, roughly corresponding to low grade squamous epithelial lesions or a worse (LSIL+) in the Bethesda 2001 reporting system. A borderline cytology (Papanicolaou class II, corresponding to atypical squamous cells of undetermined significance (ASC-US) or reactive cytological abnormality, or both, in the Bethesda 2001) usually leads to a recommendation for intensified screening, meaning a repeated screening invitation after 12-24 months. Women with negative results on colposcopy and histology are also recommended for intensified screening. If women have borderline results on cytology two or three times during intensified screening, they are referred for colposcopy. ${ }^{15}$ The threshold for treatment in the current programme is a CIN I lesion. In Finland, CIN I lesions in women aged 30 or more are treated, but women aged under 30 tend to be followed up without treatment.

\section{HPV screening protocol}

In the HPV screening protocol women underwent an HPV DNA test instead of conventional cytology as the primary screening test. After the conventional cytological smear was prepared (see above), the rest of the cellular material from the endocervical sample was used for an HPV test. ${ }^{11-14}$ The transport medium containing the screening sample was processed with the supplies and reagents of the hybrid capture II assay (Qiagen Finland, Helsinki). Results of the detection assay were expressed as a ratio of relative light units (RLU ratio) with 1.00 (equivalent to HPV DNA concentration of $1 \mathrm{pg} / \mathrm{ml}$ ) as the cut off for a positive result. The test cut off and internal quality assurance procedures were according to the manufacturer's instructions.

In women with a negative HPV result, we did not evaluate cytology and the next invitation for screening was scheduled after five years as in the cytology based programme. If the HPV result was positive, cytology was evaluated with a triage method to decide about the management. The cytotechnician and thereafter the cytopathologist carried out the cytology triage as in the conventional arm. The technician analysing the smear and the physicians at the clinical phase were aware of the result of HPV DNA test. The treatment threshold was the same as for conventional screening.

In the HPV screening protocol, any decision for immediate referral was based on a clearly positive cytological finding. In cases of borderline cytology, 
intensified screening was recommended. Intensified screening was also recommended if the HPV result was positive but the result of cytological triage was negative. During the intensified screening in the HPV arm, women were referred for colposcopy after repeated borderline findings at cytological triage or after three consecutive positive HPV test results even if cytology was normal.

A small group of women, about $8.5 \%$, in the HPV arm underwent a conventional cervical smear test because an HPV test was not taken, mainly for technical reasons (the proper brush or tube was missing or the sample was not taken because of error). Few women refused the HPV test. The HPV test was not available to women in the conventional screening arm, even though in a few cases $(<0.1 \%$ of visits $)$ it was carried out in error.

\section{Follow-up data and linkage}

The screening database was linked with the population register and cancer register to obtain information on vital status, emigration, cervical cancer, cervical intraepithelial neoplasia grade III (CIN III), and adenocarcinoma in situ. All the outcomes in our study were based on data from the cancer registry. Within the studied municipalities, all screening invitations and visits were recorded. Many women were also screened outside the screening programme. Each year about the same number of cervical smears are taken opportunistically as in the organised programme, but these data are not registered. ${ }^{1516}$

The follow-up started at the time of the first valid invitation of the current randomised study period (2003-5) and closed at emigration, death, diagnosis of CIN III/adenocarcinoma in situ, or cervical cancer or at 31 December 2007, whichever was earlier. The screening data did not include the exact date of invitation, so we used the month and year of the screening visit to approximate invitation time among attendees. Among non-attendees we estimated the month of invitation as the median month of visits in their municipality during the invitational year.

\section{Sample size}

The a priori planned outcome included the incidence of invasive cervical cancer and CIN III/adenocarcinoma in situ at 5, 10, and 15 years through record linkage between screening and cancer registers. We needed about a million woman years at risk for an $80 \%$ power to detect a $50 \%$ decrease in the subsequent risk of cervical cancer. ${ }^{12}$ Considering data on CIN III+ the statistical power to detect a 1.5 -fold difference among all invitees was about $70 \%$ (relative rate $1.5, \alpha$ 0.05 , one sided) and for detecting a twofold difference among those with positive results was 95\% (2.0, $\alpha 0.05$, one sided) in current phase of follow-up.

\section{Definitions of summary screening findings}

We used the following terms: invitee-a woman who got an invitation (a personal letter) to attend screening; attendee - a woman who took part in screening (that is, a cervical sample was taken); and non-attendee-a woman who was invited for screening but did not attend.

In attendees, the main groups consisted of women with positive or negative results on primary screening tests. An RLU ratio $<1.00$ on an HPV test and Papanicolaou class I on a cervical smear test were classed as negative results. An RLU ratio $\geq 1.00$ on an HPV test or an abnormal result of the cervical smear was classed as a positive result. Women with a positive result were further grouped into those with a positive screening episode and those who were recommended for intensified screening.

Women with a positive screening episode were referred for colposcopy and thereafter any histologically confirmed cervical intraepithelial neoplasia or cervical cancer was detected at the index screening round. Women were recommended for intensified screening if the results of colposcopy or histology were negative (that is, no cervical intraepithelial neoplasia diagnosed at the index episode among recommended women) or if the result of the cervical smear was class II (in conventional screening) or the cytology triage result was normal or gave a borderline result (in HPV screening).

\section{Statistical methods}

We compared patterns of CIN III and respective sensitivities between arms with Poisson regression; the numerator was the number of detected lesions of interest, and the denominator was the woman years at risk in all women who were invited or attended. We calculated relative risk estimates (relative rate) for the HPV screening arm using the conventional screening arm as the reference. The 95\% confidence intervals for the relative risk estimates and potential effect modification with age were assessed with likelihood ratio statistics. The analyses were based on the intention to screen principle throughout, both among invitees as well as in more detailed subgroups of attendees.

The invitees were further grouped into attendees and non-attendees and the attendees into those with positive and negative test results during the first or "index" screening round (see the definitions above). Women remained in the same category throughout the current follow-up period.

\section{RESULTS}

In the 58076 women with analysed data, there were 191218 woman years at risk. The average follow-up was 3.3 years with a maximum of five years. In the HPV screening arm, 29037 women were invited and 19449 attended. In the conventional arm 29039 women were invited and 19221 attended. The woman years at risk among attendees comprised $67.0 \%$ of the person time in the HPV screening arm and $66.3 \%$ in the conventional arm (fig 1 and table 1). There were no marked differences in attendance or follow-up time between study arms. There were more women with a positive screening episode (that is, with 
Table 1 Number of women and woman years at risk with percentage distribution in cervical screening programme

\begin{tabular}{|c|c|c|c|c|c|c|c|c|}
\hline & \multicolumn{4}{|c|}{ HPV screening } & \multicolumn{4}{|c|}{ Conventional screening } \\
\hline & Women & $\begin{array}{l}\text { Woman } \\
\text { years }\end{array}$ & $\begin{array}{c}\% \text { of women } \\
\text { years }\end{array}$ & $\begin{array}{c}\text { Women years } \\
\text { per woman }\end{array}$ & Women & $\begin{array}{l}\text { Woman } \\
\text { years }\end{array}$ & $\begin{array}{c}\% \text { of women } \\
\text { years }\end{array}$ & $\begin{array}{c}\text { Women years } \\
\text { per woman }\end{array}$ \\
\hline Invitees & 29037 & 95553 & 100.0 & 3.3 & 29039 & 95666 & 100.0 & 3.3 \\
\hline Attendees & 19449 & 64025 & 67.0 & 3.3 & 19221 & 63396 & 66.3 & 3.3 \\
\hline Non-attendees & 9588 & 31528 & 33.0 & 3.3 & 9818 & 32270 & 33.7 & 3.3 \\
\hline \multicolumn{9}{|l|}{ Screening group (among attendees): } \\
\hline Screening test positive & 1354 & 4544 & 7.1 & 3.4 & 1125 & 3766 & 5.9 & 3.3 \\
\hline Screening episode positive & 110 & 370 & 0.6 & 3.4 & 72 & 235 & 0.4 & 3.3 \\
\hline Recommendation for intensified screening* & $1244 \dagger$ & 4174 & 6.5 & 3.4 & 1053 & 3531 & 5.6 & 3.4 \\
\hline Screening test negative & 18095 & 59480 & 92.9 & 3.3 & 18096 & 59630 & 94.1 & 3.3 \\
\hline \multicolumn{9}{|l|}{ By age group (years): } \\
\hline \multicolumn{9}{|l|}{ Age 30-39: } \\
\hline Invitees & 7939 & 25961 & 100.0 & 3.3 & 7894 & 25852 & 100.0 & 3.3 \\
\hline Attendees & 4630 & 15152 & 58.4 & 3.3 & 4571 & 15000 & 58.0 & 3.3 \\
\hline \multicolumn{9}{|l|}{ Age 40-49: } \\
\hline Invitees & 8893 & 29337 & 100.0 & 3.3 & 8849 & 29292 & 100.0 & 3.3 \\
\hline Attendees & 5894 & 19485 & 66.4 & 3.3 & 5728 & 18951 & 64.7 & 3.3 \\
\hline \multicolumn{9}{|l|}{ Age 50-64: } \\
\hline Invitees & 12205 & 40257 & 100.0 & 3.3 & 12296 & 40522 & 100.0 & 3.3 \\
\hline Attendees & 8925 & 29389 & 73.0 & 3.3 & 8922 & 29445 & 72.7 & 3.3 \\
\hline
\end{tabular}

*In women recommended for intensified screening, four in HPV group and 29 in conventional group had inadequate screening test.

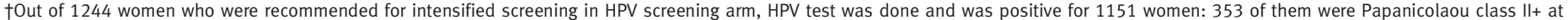
cytology triage, 794 were class I, and 4 had inadequate smear. HPV test was not done for 93 women, and recommendation was based on Papanicolaou class II+ result among them.

any diagnosis of cervical intraepithelial neoplasia or cervical cancer) in the HPV screening arm than in the conventional screening arm (110 v 72) and more recommended for intensified screening at the index screen (1244 v 1053).

There were 76 cases of CIN III+ in women invited in the HPV arm and 53 cases in those invited in the conventional arm (table 2). Figure 2 shows the cumulative
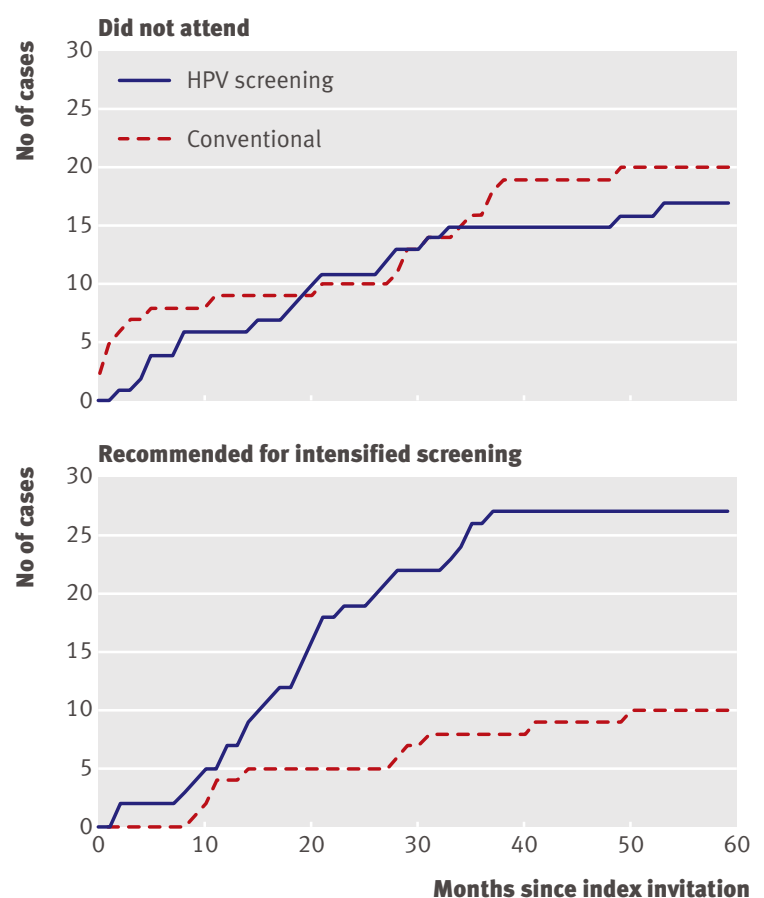

number of cases of CIN III+ over follow-up. Figure 3 shows the corresponding cumulative incidence. Compared with the conventional arm, the relative rate of CIN III+ in the HPV arm was 1.44 (95\% confidence interval 1.01 to 2.05 ; table 2 ) in all women invited. In attendees there were 59 cases of CIN III+ lesions in the HPV arm and 33 in the conventional arm $(1.77,1.16$ to 2.74). The relative rate of cervical cancer in the HPV
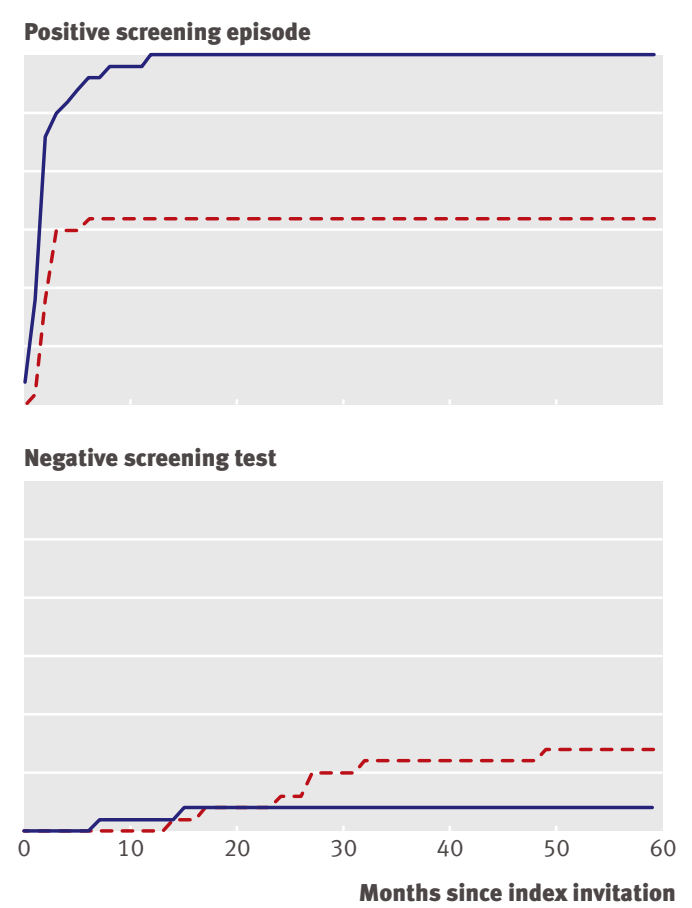
Table 2 | Numbers of cases of cervical cancer, adenocarcinoma in situ, and CIN III and relative rate $(95 \%$ confidence interval) by study arm for all women who were invited for cervical screening and those who attended

\begin{tabular}{|c|c|c|c|}
\hline \multirow[b]{2}{*}{ Study group } & \multicolumn{2}{|c|}{ No of cases } & \multirow[b]{2}{*}{$\begin{array}{c}\mathrm{RR}(95 \% \mathrm{Cl}) \text { for comparison } \\
\text { between arms }\end{array}$} \\
\hline & $\begin{array}{c}\text { HPV } \\
\text { screening }\end{array}$ & $\begin{array}{l}\text { Conventional } \\
\text { screening }\end{array}$ & \\
\hline \multicolumn{4}{|c|}{ Cervical cancer, adenocarcinoma in situ, or CIN III } \\
\hline Invitees & 76 & 53 & 1.44 (1.01 to 2.05$)$ \\
\hline Attendees & 59 & 33 & 1.77 (1.16 to 2.74$)$ \\
\hline \multicolumn{4}{|l|}{ Cervical cancer } \\
\hline Invitees & 6 & 8 & $0.75(0.25$ to 2.16$)$ \\
\hline Attendees & 6 & 3 & $1.98(0.52$ to 9.38$)$ \\
\hline \multicolumn{4}{|c|}{ Adenocarcinoma in situ } \\
\hline Invitees & 7 & 5 & $1.40(0.44$ to 4.73$)$ \\
\hline Attendees & 5 & 3 & $1.65(0.40$ to 8.04$)$ \\
\hline \multicolumn{4}{|l|}{ CIN III } \\
\hline Invitees & 63 & 40 & 1.58 (1.07 to 2.36$)$ \\
\hline Attendees & 48 & 27 & 1.76 (1.11 to 2.86$)$ \\
\hline
\end{tabular}

arm compared with the conventional arm was 0.75 (0.25 to 2.16) in invitees and 1.98 (0.52 to 9.38) in attendees, based on six and eight cases in invitees and six and three cases in attendees.

Table 3 gives information on CIN III+ by main groups at index screening. There was an increase in the detection of cases of CIN III+ in the HPV arm in both groups of women with positive screening test results - that is, those with a positive episode and those with a recommendation for intensified screening. In women who had a negative screening test result the

Table $3 \mid$ Number of cases of cervical cancer, adenocarcinoma in situ, or CIN III with relative rate $(95 \%$ confidence interval) by study arm and screening result among women who attended cervical screening programme

\begin{tabular}{|c|c|c|c|}
\hline \multirow[b]{2}{*}{ Study group } & \multicolumn{2}{|c|}{ No of cases } & \multirow{2}{*}{$\begin{array}{c}\mathrm{RR}(95 \% \mathrm{Cl}) \\
\text { for comparison } \\
\text { between arms }\end{array}$} \\
\hline & HPV screening & $\begin{array}{l}\text { Conventional } \\
\text { screening }\end{array}$ & \\
\hline \multicolumn{4}{|l|}{ Cervical cancer, adenocarcinoma in situ, or CIN III } \\
\hline Screening test positive & 57 & 26 & 2.17 (1.38 to 3.51$)$ \\
\hline Screening episode positive & 30 & 16 & $1.86(1.03$ to 3.49$)$ \\
\hline Recommendation for intensified screening & 27 & 10 & $2.67(1.34$ to 5.80$)$ \\
\hline Screening test negative & 2 & 7 & $0.28(0.04$ to 1.17$)$ \\
\hline \multicolumn{4}{|l|}{ Cervical cancer } \\
\hline Screening test positive & 5 & 3 & $1.65(0.40$ to 8.04$)$ \\
\hline Screening episode positive & 3 & 2 & $1.49(0.25$ to 11.3$)$ \\
\hline Recommendation for intensified screening & 2 & 1 & $1.98(0.19$ to 42.6$)$ \\
\hline Screening test negative & 1 & 0 & NA \\
\hline \multicolumn{4}{|l|}{ Adenocarcinoma in situ } \\
\hline Screening test positive & 5 & 2 & $2.48(0.53$ to 17.3$)$ \\
\hline Screening episode positive & 0 & 1 & $0.00(\mathrm{NA})$ \\
\hline Recommendation for intensified screening & 5 & 1 & $4.95(0.80$ to 94.8$)$ \\
\hline Screening test negative & 0 & 1 & 0.00 (NA) \\
\hline \multicolumn{4}{|l|}{ CIN III } \\
\hline Screening test positive & 47 & 21 & 2.22 (1.34 to 3.78$)$ \\
\hline Screening episode positive & 27 & 13 & $2.06(1.08$ to 4.12$)$ \\
\hline Recommendation for intensified screening & 20 & 8 & $2.48(1.13$ to 5.97$)$ \\
\hline Screening test negative & 1 & 6 & $0.17(0.01$ to 0.97$)$ \\
\hline
\end{tabular}

relative rate between arms was 0.28 (0.04 to 1.17$)$, suggesting a lower rate of CIN III+ for HPV screening than for conventional screening, though not significant.

In 1244 women recommended for intensified screening in the HPV arm, 794 women had a positive HPV test result but a negative result on cytology triage (Papanicolaou class I); 353 women had a positive HPV test result and at least borderline result on cytological triage; four women remained with an inadequate screening result because of inadequate cytological triage; and for 93 women cytology was done as the primary screening test. In the intensified screening in these subgroups CIN III+ was subsequently detected in 11,15 , zero, and one, respectively. These correspond to 10 cases detected in the whole conventional screening arm, all based on a borderline cytological finding.

Further analyses by age groups indicated that there was no marked variation in the patterns of CIN III+ between screening arms over age groups (table 4).

\section{DISCUSSION}

In a well established routinely organised screening programme HPV testing with cytology triage was more sensitive than conventional cytology in the detection of cervical intraepithelial neoplasia grade III and above (CIN III+). We looked at primary HPV DNA test with cytology triage compared with the conventional cervical smear test. The study was based on a maximum followup of five years since the index invitation.

Our earlier cross sectional studies on data from the screening register also suggested higher sensitivity for primary HPV screening with cytology triage compared with conventional cytology when any cervical intraepithelial neoplasia at the recruitment was used as a surrogate indicator. ${ }^{113}$ The specificity of the two testing protocols was similar. Our study adds longitudinal information based on cancer registry files and confirms a higher sensitivity of HPV screening compared with conventional screening, yielding more cases of CIN III+ within the recruitment period - that is, the "index" episode - and also in the intensified screening after a positive HPV test result. At the same time the incidence of CIN III+ among women with negative results seemed lower in the HPV arm than in the conventional arm.

\section{Strengths and limitations}

The random allocation of the two screening methods within the routine screening programme took care of the comparability problems between arms. The average attendance rate in Finland is $72 \%$, but in southern Finland, where we conducted our study, it has been constantly lower at $66 \% .{ }^{17}$ This relatively low attendance rate is still a problem, and several earlier cohort studies have shown more cervical cancer among women who do not attend screening. ${ }^{2}$ More effort is needed to improve attendance. A few more cases of cervical cancer were subsequently detected in nonattendees in the conventional arm than in the HPV arm, even though the corresponding number of 

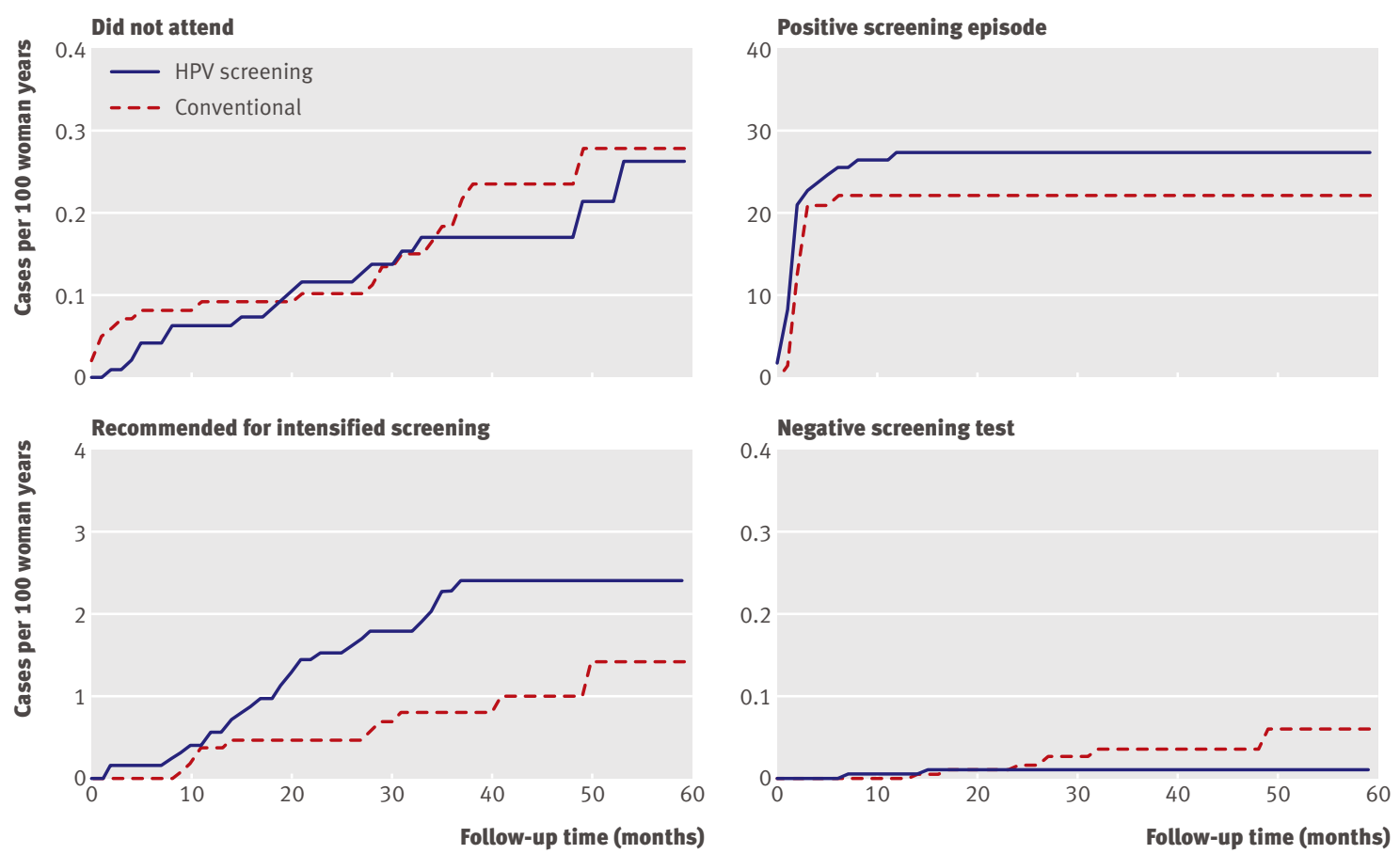

No of women in follow-up by month since index invitation:

$\begin{array}{lrccccc}\text { Did not attend } & 0 & 9 & 19 & 29 & 39 & 49 \\ \text { HPV } & 9588 & 9508 & 9441 & 7705 & 5490 & 2298 \\ \begin{array}{l}\text { Conventional } \\ \text { Positive screening episode }\end{array} & 9818 & 9739 & 9674 & 7879 & 5639 & 2330 \\ \text { HPV } & 110 & 83 & 83 & 73 & 49 & 26 \\ \begin{array}{l}\text { Conventional } \\ \text { Recommended for intensified screening }\end{array} & 72 & 56 & 56 & 50 & 34 & 15 \\ \text { HPV } & 1244 & 1239 & 1222 & 981 & 628 & 328 \\ \begin{array}{l}\text { Conventional } \\ \text { Negative screening episode }\end{array} & 1053 & 1052 & 1045 & 863 & 523 & 247 \\ \text { HPV } & 18095 & 18062 & 18015 & 14745 & 8601 & 3622 \\ \text { Conventional } & 18096 & 18057 & 18015 & 14741 & 8708 & 3759\end{array}$

Fig 3 | Cumulative incidence rates (cases per 1000 woman years) by months since invitation

CIN III+ cases was similar (fig 1). The difference was based on small numbers and thus probably caused by chance. According to the protocol, women were not informed about the method when they were invited for screening; the method was explained to them at the screening visit. There might have been differences by arm in attendees' subsequent use of healthcare services. Also, potential fluctuations in the diagnostic criteria could affect overdiagnosis of the CIN III or adenocarcinoma in situ lesions, affecting balances between benefits and harms when the tests are used in a routine programme.

\section{Comparison with other studies}

A single round of HPV testing has been shown to reduce mortality from cervical cancer as well as the number of cases of advanced cervical cancer. ${ }^{10}$ The current evidence from randomised controlled trials and longitudinal follow-up studies of CIN III+ suggests that validated HPV DNA testing with simultaneous cytology for all women is more sensitive than cytology alone and identifies more CIN III+ lesions early and fewer subsequent CIN III+ lesions after a negative HPV test result. $^{6-91819}$ The second screening round seems to equalise the two methods regarding the long term rates of pre-cancer treatment. ${ }^{78}$ Although our study was done in the routine screening service, our results are consistent with those previously reported.

Even though there was no clear indication of interaction in effect by age group, we need to take into account the additional burden of investigations caused by high rate of positive test results in the HPV screening in young women $($ age $<35) .{ }^{14}$ In screened women in Finland about $8 \%$ have a positive HPV test result; rising to $15-25 \%$ in the youngest targeted age groups. ${ }^{140}$ Cytology triage after a positive result reduces the referral rate; during the first three years, there was no difference in the referral rates between study arms and a lower rate of detection of ASCUS (atypical squamous cells of undetermined significance) in the HPV screening arm than in the conventional arm. ${ }^{14}$ This is because in conventional screening ASCUS is often observed in 
Table 4 | Numbers of cases of CIN III+, with relative rate ( $95 \%$ confidence intervals) by age group and screening result among women who attended cervical screening programme

\begin{tabular}{|c|c|c|c|}
\hline $\begin{array}{l}\text { Age group (years) at invitation, and } \\
\text { screening finding }\end{array}$ & HPV screening & Conventional & $\begin{array}{l}\mathrm{RR}(95 \% \mathrm{Cl}) \text { for comparison } \\
\text { between arms* }\end{array}$ \\
\hline \multicolumn{4}{|l|}{ Age 30-39 } \\
\hline Total & 26 & 14 & 1.84 (0.98 to 3.62$)$ \\
\hline Screening test positive & 25 & 9 & 2.75 (1.33 to 6.23$)$ \\
\hline Screening episode positive & 14 & 6 & 2.31 (0.93 to 6.52$)$ \\
\hline Screening test negative & 1 & 5 & $0.20(0.01$ to 1.24$)$ \\
\hline \multicolumn{4}{|l|}{ Age $40-64$} \\
\hline Total & 33 & 19 & 1.72 (0.99 to 3.08$)$ \\
\hline Screening test positive & 32 & 17 & $1.86(1.05$ to 3.43$)$ \\
\hline Screening episode positive & 16 & 10 & $1.58(0.73$ to 3.62$)$ \\
\hline Screening test negative & 1 & 2 & $0.50(0.02$ to 5.23$)$ \\
\hline \multicolumn{4}{|l|}{ Any age } \\
\hline Total & 59 & 33 & 1.77 (1.16 to 2.74$)$ \\
\hline Screening test positive & 57 & 26 & 2.17 (1.38 to 3.51$)$ \\
\hline Screening episode positive & 30 & 16 & 1.86 (1.03 to 3.49$)$ \\
\hline Screening test negative & 2 & 7 & 0.28 (0.04 to 1.17$)$ \\
\hline
\end{tabular}

*For effect modification between age group and screening arm, $\mathrm{P}=0.88$ in all attended; $\mathrm{P}=0.55$ for positive screening episode; $\mathrm{P}=0.42$ for positive screening test result; $\mathrm{P}=0.58$ for negative screening test result.

women who are not positive for HPV. In our study, many women underwent intensified screening because of a positive HPV test result. One option would be to base the recommendation for intensified screening on the results of cytology triage alone. In our study we could not have improved specificity like this without a considerable loss in sensitivity. It seems justified to conclude that in the youngest age groups better triage tests are still needed and screening women aged $<35$ with the primary HPV test is challenging because of the high probability of a positive result.

\section{Policy implications}

Considering the high probability of progression of CIN III lesions in women aged $\geq 35,{ }^{5}$ our results are important for prevention of cervical cancer. Compared with conventional screening in this older age group, the burden to women of re-testing, referral, and treatment because of a positive result of a primary screening test does not seem too high with HPV screening. ${ }^{14}$ One problem is that HPV screening can more often lead to detection of lesions with lower potential for progression (CIN I and II) and at an earlier age, ${ }^{781418}$ thus potentially affecting quality of life. In the future, a new policy with an increased interval between screenings could provide a solution.

\section{WHAT IS ALREADY KNOWN ON THIS TOPIC}

Primary HPV screening with simultaneous cytology is more sensitive in detecting cervical cancer and pre-cancerous lesions than screening with conventional cytology alone

\section{WHAT THIS STUDY ADDS}

This longitudinal study showed that primary HPV screening with cytology triage in a routine organised screening programme was more sensitive than conventional cytology in detecting cervical cancer and severe pre-cancerous lesions in all age groups
Our study had limited statistical power to analyse any impact on invasive cervical cancer. Additionally, the power to observe differences among women with negative test results was not satisfactory. To reach optimal information not only on the effectiveness of primary HPV screening based programme but also on performance and screening policies (age groups and screening intervals), we need to continue the randomised screening protocol at least for an entire followup screening round - that is, up to 10 years after the introduction of the study. ${ }^{12}$ Our intention is to rescreen women according to the same allocation at least twice.

Primary HPV screening should be piloted in an organised programme, and extension towards a national implementation should be considered if the outcome is favourable. ${ }^{4}$ As characteristics and organisational details between cervical cancer screening programmes are highly variable in different countries, each programme needs to evaluate the new methods in their own context. Our study integrates a rigorous evaluation design with running the pilot in the Finnish screening programme. We think that gradual implementation of HPV screening in regions other than those in this trial is justified.

We thank the staff of the Mass Screening Registry and the Finnish Cancer Registry, the Pathology Laboratory of the Finnish Cancer Society in Helsinki, and the HUSLAB Kätilöopisto Pathology Laboratory, Helsinki, for their valuable contribution throughout the study.

Contributors: $\mathrm{AA}, \mathrm{MH}$, and PN were involved in the planning, design, data collection, analyses, and reporting. LK-T and ML contributed to the coordination of the trial and the data collection, analysis, and reporting. $\mathrm{NM}, \mathrm{JT}$, and PL have contributed to data collection, analysis, and reporting. PN is guarantor.

Funding: The study was partially financed by grants from the European Commission, Europe Against Cancer action programme through European Cervical Cancer Screening Network; the Academy of Finland; and the Cancer Organisation of Finland.

Competing interests: All authors have completed the Unified Competing Interest form at www.icmje.org/coi_disclosure.pdf (available on request from the corresponding author) and declare that all authors had: (1) No financial support for the submitted work from anyone other than their employer; (2) No financial relationships with commercial entities that might have an interest in the submitted work; (3) No spouses, partners, or children with relationships with commercial entities that might have an interest in the submitted work; (4) No Non-financial interests that may be relevant to the submitted work.

Ethical approval: The protocol for the randomised screening study was approved by the ethical committee of the National Research and

Development Centre for Welfare and Health (STAKES, 4151/54/98), the ethical committee of the Obstetrics and Gynaecology in Hospital District of Helsinki and Uusimaa (221/E8/02), and the National Authority for Medicolegal Affairs (3950/32/300/02). Every woman was informed about the test and could refuse the assigned HPV test and receive the conventional test instead.

Data sharing: No additional data available.

1 Screening for cancer of the uterine cervix. From the IARC working group on cervical cancer screening and the UICC project group on the evaluation of screening programmes for cancer. IARC Sci Publ 1986;76:1-315.

2 International Agency for Research on Cancer. IARC/WHO handbooks of cancer prevention. Vol 10: cervix cancer screening. IARC Press, 2005.

3 International Agency for Research on Cancer. IARC monographs on the evaluation of carcinogenic risks to humans. Vol 90: human papillomaviruses. IARC Press, 2007.

4 Arbyn M, Dillner J, Schenck U, Nieminen P, Weiderpass E, Da Silva ID, et al. Methods for screening and diagnosis. In: Arbyn M, Anttila A, Jordan J, Ronco G, Segnan N, Wiener HG, eds. European guidelines 
for quality assurance in cervical cancer screening. 2nd ed. Office for Official Publications of the European Communities, 2008.

5 Van Oortmarssen GJ, Habbema JD. Epidemiological evidence for age dependent regression of pre-invasive cervical cancer. Br J Cancer 1991;64:559-65.

6 Khan MJ, Castle PE, Lorincz AT, Wacholder S, Sherman M, Scott DR, et al. The elevated 10-year risk of cervical precancer and cancer in women with human papillomavirus (HPV) type 16 or 18 and the possible utility of type-specific HPV testing in clinical practice. / Nat Cancer Inst 2005;97:1072-9.

7 Bulkmans NWJ, Berkhof J, Rozendaal L, van Kemenade FJ, Boeke AJP, Bulk S, et al. Human papillomavirus DNA testing for the detection of cervical intraepithelial neoplasia grade 3 and cancer: 5 -year followup of a randomised controlled implementation trial. Lancet 2007:370:1764-72.

8 Naucler P, Ryd W, Törnberg S, Strand A, Wadell G, Elfgren K, et al. Human papillomavirus and papanicolaou tests to screen for cervical cancer. N Engl J Med 2007;357:1589-97.

9 Dillner J, Rebolj M, Birembaut P, Petry K-U, Szarewski A, Munk C, et al. Long term predictive values of cytology and human papillomavirus testing in cervical cancer screening: joint European cohort study. BM 2008;377:a1754.

10 Sankaranarayanan R, Nene BM, Shastri SS, Jayant K, Muwonge R, Budukh AM, et al. HPV screening for cervical cancer in rural India. $N$ Engl J Med 2009;360:1385-94.

11 Kotaniemi-Talonen L, Nieminen P, Anttila A, Hakama M. Routine cervical screening with primary HPV testing and cytology triage protocol in a randomized setting. Br J Cancer 2005;93:862-7.

12 Anttila A, Hakama M, Kotaniemi-Talonen L, Nieminen P. Alternative technologies in cervical cancer screening: a randomised evaluation trial. BMC Public Health 2006;6:252.

13 Kotaniemi-Talonen L, Anttila A, Malila N, Tarkkanen J, Laurila P, Hakama M, et al. Screening with a primary human papillomavirus test does not increase detection of cervical cancer and intraepithelial neoplasia 3. Eur J Cancer 2008;44:565-71.

14 Leinonen M, Nieminen P, Kotaniemi-Talonen L, Malila N, Hakama M, Tarkkanen J, et al. Age-specific performance of cervical cancer screening with primary HPV DNA test compared to conventional cytological screening in a randomised setting. J Natl Cancer Inst 2009;101:1612-23.

15 Anttila A, Nieminen P. Cervical cancer screening programme in Finland. Eur / Cancer 2000;36:2209-14.

16 Monto K, Nieminen P. Gynekologisten irtosolunäytteiden määrät ja raportointitavat Suomessa [Finnish]. Suomen Lääkärilehti 2005;60:2093-7.

17 Finnish Cancer Registry-Institute for Statistical and Epidemiological Cancer Research. Cancer in Finland 2006 and 2007. Cancer Statistics of the National Institute for Health and Welfare (THL). Cancer Society of Finland Publication No. 76, Helsinki 2009.

18 Ronco G, Giorgi-Rossi P, Carozzi F, Confortini M, Dalla Palma P, Del Mistro $\mathrm{A}$, et al. Results at recruitment from a randomized controlled trial comparing human papillomavirus testing alone with conventional cytology as the primary cervical cancer screening test. Natl Cancer Inst 2008;100:492-501.

19 Kitchener HC, Almonte M, Thomson C, Wheeler P, Sargent A, Stoykova B, et al. HPV testing in combination with liquid-based cytology in primary cervical screening (ARTISTIC): a randomised controlled trial. Lancet Oncol 2009;10:672-82.

20 Leinonen M, Kotaniemi-Talonen L, Anttila A, Dyba T, Tarkkanen J, Nieminen P. Prevalence of oncogenic human papillomavirus infection in an organised screening population in Finland. Int / Cancer 2008;123:1344-9.

Accepted: 11 January 2010 